\title{
Kernel Based Telegraph-Diffusion Equation for Image Noise Removal
}

\author{
Yu-Qian Yang ${ }^{1}$ and Cheng-Yi Zhang ${ }^{2}$ \\ ${ }^{1}$ School of Mathematics and Statistics, Xidian University, Xian, Shaanxi 710075, China \\ ${ }^{2}$ Department of Mathematics and Mechanics of School of Science, Xian Polytechnic University, Xian, Shaanxi 710048, China \\ Correspondence should be addressed to Yu-Qian Yang; yqyang@xidian.edu.cn
}

Received 10 March 2014; Revised 7 May 2014; Accepted 7 May 2014; Published 22 May 2014

Academic Editor: Ebrahim Momoniat

Copyright (C) 2014 Y.-Q. Yang and C.-Y. Zhang. This is an open access article distributed under the Creative Commons Attribution License, which permits unrestricted use, distribution, and reproduction in any medium, provided the original work is properly cited.

\begin{abstract}
The second-order partial differential equations have good performances on noise smoothing and edge preservation. However, for low signal-to-noise ratio (SNR) images, the discrimination between edges and noise is a challenging problem. In this paper, the authors propose a kernel based telegraph-diffusion equation (KTDE) for noise removal. In this method, a kernelized gradient operator is introduced in the second-order telegraph-diffusion equation (TDE), which leads to more effective noise removal capability. Experiment results show that this method outperforms several anisotropic diffusion methods and the TDE method for noise removal and edge preservation.
\end{abstract}

\section{Introduction}

Noise removal is an important step in image processing, but there is a tradeoff between noise removal and edge preservation. Different adaptive methods are needed for this object. In the last two decades, the partial differential equation (PDE) methods have been broadly used in image noise removal. These methods include anisotropic diffusion equations [1] and total variation models [2] as well as curve evolution equations [3]. The first kind of these methods called anisotropic diffusion is introduced by Perona and Malik [1] in which the denoised image is the solution of a nonlinear second-order PDE. Let $u$ denote the image intensity function, $c(\cdot)$ the diffusion coefficient, and $t$ the time; then the PM diffusion model is

$$
\frac{\partial u}{\partial t}-\nabla \cdot(c(\nabla u) \nabla u)=0,
$$

where $\nabla$. and $\nabla$ denote the divergence and the gradient, respectively. Since then a variety of nonlinear diffusion denoising techniques were proposed [4-10]. But the blocky effects can be caused by second-order PDE. Then some fourth-order anisotropic diffusion methods were also proposed for the blocky effect elimination [11-16], which replace the gradient operator in second-order PDE with a Laplace operator. In [17], a telegraph-diffusion model was proposed, which can give more sharp edges than other PDEs based models. In [18], a fourth-order telegraph-diffusion was proposed for image denoising and edge preservation. These PDE methods can distinguish edge points and noise points by utilizing a gradient operator and iteratively eliminate the noise in the diffusion process. They respect region boundaries or small structures, so these techniques can simultaneously eliminate noise and preserve or even enhance edges. Although these methods can restore images with discontinuities, they cannot give outstanding performance on low SNR images. Many images such as medical images and natural images are corrupted by heavy noise. In these images, noise variations may be comparable to or even greater than edge variations, so it is difficult to produce satisfactory results using conventional PDE methods. The gradient operator cannot achieve an effective separation of edges and noise.

In this paper, a kernel based telegraph-diffusion equation (KTDE) is proposed. There are two primary advantages of this new method. First, we use the telegraph-diffusion model proposed by Ratner and Zeevi [17], which leaves much more sharp edges than other diffusion methods. Second, kernel function is incorporated into the partial differential equation 
due to its better signal/noise separation ability. It maps the input space to a higher-order feature space, in which the discrimination of edges and noise becomes a linear problem. The proposed method has been applied to natural images, and experiment results outperform those produced by original PM diffusion and other improved models.

The remainder of this paper is organized as follows. Section 2 introduces the telegraph-diffusion filter and the concept of the kernel method. Section 3 describes the proposed kernel based telegraph-diffusion method. The numerical implementation of the proposed method is given in this section. The experiment results are compared with other diffusion methods in Section 4. Section 5 concludes this paper.

\section{Telegraph-Diffusion and Kernel Method}

2.1. Telegraph Diffusion. The nonlinear diffusion filter proposed by Perona and Malik [1] is given by

$$
\frac{\partial u}{\partial t}=\nabla \cdot(c(\|\nabla u\|) \nabla u)
$$

where $u$ is the noisy image, $t$ is the evolution time, and $c(\cdot)$ is a diffusivity function, which decides the degree of denoising and preservation of singularities, for example, noise and edges. The diffusivity function is a positive and nonincreasing function of $\|\nabla u\|$. Perona and Malik suggested two diffusivity functions:

$$
\begin{gathered}
c(\|\nabla u\|)=\frac{1}{1+(\|\nabla u\| / k)^{2}}, \\
c(\|\nabla u\|)=\exp \left[-\left(\frac{\|\nabla u\|}{k}\right)^{2}\right],
\end{gathered}
$$

where $k$ is the so-called contrast parameter. If $\|\nabla u\| \gg k$, $c(\|\nabla u\|) \rightarrow 0$, then the diffusion is suppressed; if $\|\nabla u\| \ll k$, $c(\|\nabla u\|) \rightarrow 1$, then the diffusion is encouraged and noise is smoothed. In general, a large value of $k$ leads to a smoother result in homogeneous regions than a smaller one. It is known that the solution of (2) is equal to the minimization of the energy function $[19,20]$ :

$$
E(u)=\int_{\Omega} f(\|\nabla u\|) d \Omega,
$$

where $\Omega$ is the image domain and $f(\cdot)$ is an increasing nonnegative function with

$$
c(s)=\frac{f^{\prime}(s)}{s},
$$

and $E(u)$ is minimized leading to a piecewise constant approximation of $u$. Recently, a new approach called telegraphdiffusion model has been presented in [17]

$$
\frac{\partial^{2} u}{\partial t^{2}}+\lambda \frac{\partial u}{\partial t}-\nabla \cdot(c(\|\nabla u\|) \nabla u)=0,
$$

where $\lambda$ is the damping coefficient. It is a parabolic-hyperbolic equation and it can create denoising and edge preserving effect. The TDE model is derived from PM model by adding second time derivative of the image, which can effectively eliminate the diffusion effect in the vicinity of edges and some important textures to some degree. So this model leaves more sharp edges. When the noise is large, (7) will be unstable which is similar to that of the PM model. To overcome this problem, an improved TDE (ITDE) method was proposed in [21]

$$
\frac{\partial^{2} u}{\partial t^{2}}+\lambda \frac{\partial u}{\partial t}-\nabla \cdot\left(g\left(\left|\left(\nabla G_{\sigma}\right) * u\right|\right) \nabla u\right)=0,
$$

where $G_{\sigma}$ is a Gaussian filter with standard deviation $\sigma$. In [21] Cao et al. gave the proof of the existence and uniqueness of weak solutions of model (8). As they pointed out, most of the evolution equations on image restoration are parabolic equations. But hyperbolic PDEs could improve the quality of the detected edges and so enhance the images better than parabolic PDEs. Equations (7) and (8) inherit this merit in edge preservation and image denoising. So they have better performance for human vision. But when the SNR is low, they cannot give satisfactory results.

2.2. The Kernel Based Diffusion Method. For low SNR images, the separation of edges and noise can be viewed as a nonlinearly separable classification problem. Yu et al. [22]. suggested mapping the intensity values to a higher-dimensional reproducing kernel Hilbert space (RKHS) to make their differences more discriminant for edge and noise separation. The kernel method is applied in second-order diffusion filter and the resulting filter is called kernel anisotropic diffusion (KAD) filter. In [23], a regularized gradient kernel anisotropic diffusion is proposed for image filtering.

Suppose that the input data is represented by $X(X \subseteq R)$, and $F\left(F \subseteq R^{n}\right)$ is a feature space associated with $X$ by a nonlinear mapping function $\Phi$ :

$$
\Phi: X \longrightarrow F, \quad x \longrightarrow \Phi(x),
$$

where $x$ is an input in $X$, which is mapped to a feature space with dimensionality of $n$. So the nonlinear separable classes in the original space can be linearly separated. In most cases, the map $\Phi$ cannot be found. However, Mercer kernels induce an inner product in the high-dimensional space under the mapping $\Phi$ :

$$
K(X, Y)=\Phi(X)^{T} \Phi(Y) .
$$

Thus kernel methods compute the inner product of two input vectors in feature space $F$ without having to explicitly compute the mapping function, nor the inner product in $F$. Methods that use dot products can be converted to the kernel methods that use kernel functions for pattern classification. The kernel methods can efficiently represent complicated nonlinear relations of the input data and retain computational simplicity. Commonly used kernel functions are polynomial kernels, radial-basis function kernels:

$$
\begin{gathered}
K(x, y)=\left(x^{T} y+1\right)^{d}, \\
K(x, y)=\exp \left(-\frac{\|x-y\|^{2}}{2 \sigma^{2}}\right) .
\end{gathered}
$$


The proposed kernel anisotropic diffusion (KAD) substitutes the gradient magnitude $\|\nabla u\|$ in the PM model with $\|\nabla \Phi(u)\|$, and then the anisotropic diffusion becomes

$$
\frac{\partial u}{\partial t}=\operatorname{div}[c(\nabla(\|\Phi(u)\|)) \nabla u]
$$

where $\|\nabla[\Phi(u)]\|$ can be calculated as

$$
\begin{array}{r}
\|\nabla[\Phi(u)]\|_{p}=\left[\frac { 1 } { | \xi _ { p } | } \sum _ { q \in \xi _ { p } } \left(K\left(u_{p}, u_{p}\right)+K\left(u_{q}, u_{q}\right)\right.\right. \\
\left.\left.-2 K\left(u_{p}, u_{q}\right)\right)\right]^{0.5},
\end{array}
$$

where $\xi_{p}$ is the spatial neighborhood of pixel $p$ and $\left|\xi_{p}\right|$ is the cardinality of $\xi_{p}$. Usually eight neighboring pixels are selected around $p$, so the cardinality of $\xi_{p}$ is 8 . If we use the radial-basis function kernel $K(X, Y)=\exp \left(-|X-Y|^{2} / 2 \sigma^{2}\right)$ in which $K(X, X)=K(Y, Y)=1,\|\nabla[\Phi(u)]\|_{p}$ becomes

$$
\|\nabla[\Phi(u)]\|_{p}=\left[\frac{1}{\left|\xi_{p}\right|} \sum_{q \in \xi_{p}}\left(2-2 K\left(u_{p}, u_{q}\right)\right)\right]^{0.5} .
$$

In contrast to using the kernel function in anisotropic diffusion method, we propose to apply the kernel function in the telegraph-diffusion method for noise removal. Our motivation is based on the separation ability of the kernel function for low SNR images.

\section{The Proposed Method}

3.1. Kernel Based Telegraph-Diffusion Equation. Inspired by the merit of kernel method and telegraph-diffusion equation, we propose the following kernel based telegraph-diffusion equation (KTDE):

$$
\frac{\partial^{2} u}{\partial t^{2}}+\lambda \frac{\partial u}{\partial t}-\nabla \cdot(c(\|\nabla(\Phi(u))\|) \nabla u)=0,
$$

where $\lambda>0$ is a damping coefficient. The proposed method is derived from the KAD method by adding second time derivative. The diffusivity function is selected as

$$
\begin{aligned}
c(\|\nabla(\Phi(u))\|) & =\frac{1}{1+(\|\nabla(\Phi(u))\| / k)^{2}}, \\
c(\|\nabla(\Phi(u))\|) & =\exp \left[-\left(\frac{\|\nabla(\Phi(u))\|}{k}\right)^{2}\right], \\
\|\nabla[\Phi(u)]\|_{p} & =\left[\frac{1}{\left|\xi_{p}\right|} \sum_{q \in \xi_{p}}\left(2-2 K\left(u_{p}, u_{q}\right)\right)\right]^{0.5},
\end{aligned}
$$

where $\xi_{p}$ represents the spatial neighborhood of pixel $p$ (we select eight neighboring pixels around $p$ ), $\left|\xi_{p}\right|$ is the cardinality of $\xi_{p}$, and $K(\cdot, \cdot)$ is the radial-basis function. As proposed in [22], the linearly separable relationships between edges and noise no longer exist in low SNR images, and the telegraph-diffusion equation can be improved by introducing a more effective edges/noise separation technique to substitute the gradient operator. The kernel function is used to map the input space to a higher-order feature space and then discriminate between edges and noise in the feature space because of its capabilities in nonlinear pattern representation. In the proposed method, the $\|\nabla[\Phi(u)]\|$ is the gradient magnitude in the feature space, so it can separate edges and noise effectively.

In this diffusion filter, there are two parameters that should be estimated: the edge detection threshold and the stopping time. For edge detection threshold $k$, we use the median absolute deviation which is adjusted for kernel anisotropic diffusion in [22]

$$
k=c \cdot \operatorname{MAD}(\|\nabla(\Phi(u))\|),
$$

where $c=1.4826$ normalizes the MAD to that of normal distribution with zero-mean and unit variance. $\operatorname{MAD}(\|\nabla(\Phi(u))\|)=\operatorname{median}[\mid\|\nabla(\Phi(u))\| \quad-$ $\operatorname{median}(\|\nabla(\Phi(u))\|) \mid]$.

In diffusion methods, it is common to terminate the diffusion after a fixed number of diffusion iterations. This mechanism is not flexible and it is difficult to produce satisfactory results. We utilize the mean absolute error criterion [24] to stop the diffusion. The MAE between two adjacent diffusion steps can be written as

$$
\operatorname{MAE}\left(u^{t}\right)=\frac{1}{M \times N} \sum_{i=1, j=1}^{i=M, j=N} \sqrt{\left(u_{i, j}^{t}-u_{i, j}^{t-1}\right)^{2}}
$$

where $u_{i, j}^{t}$ and $u_{i, j}^{t-1}$ are the filtered values of the pixels $(i, j)$ at iterations $t$ and $t-1$, respectively. $M$ and $N$ are the numbers of rows and columns of the images. When the MAE is small enough, it means that the difference between two iterations is very small and the diffusion should be terminated.

3.2. The Implementation of This Model. The processing of a digital image requires a discrete realization of the proposed filter. Equation (16) is solved numerically using an iterative approach. We construct an explicit discrete scheme to numerically solve differential (16). The time step size is $\Delta t$ and a space grid size is $h$. We quantize

$$
\begin{aligned}
& x=i h, \quad i=0,1,2, \ldots, N \\
& y=j h, \quad j=0,1,2, \ldots, M \\
& t=n \Delta t, \quad n=0,1,2, \ldots,
\end{aligned}
$$

where $M \times N$ is the size of the image. First, the symmetric boundary conditions are given as follows:

$$
\begin{array}{ll}
u_{-1, j}^{n}=u_{0, j}^{n}, & u_{N+1, j}^{n}=u_{N, j}^{n}, \quad j=0,1,2, \ldots, M, \\
u_{i,-1}^{n}=u_{i, 0}, & u_{i, M+1}^{n}=u_{i, M}^{n}, \quad i=0,1,2, \ldots, N .
\end{array}
$$


Second, we use the following discrete approximation:

$$
\begin{gathered}
\nabla_{x}^{+} u_{i, j}^{n}=\frac{u_{i+1, j}^{n}-u_{i, j}^{n}}{h}, \quad \nabla_{x}^{-} u_{i, j}^{n}=\frac{u_{i-1, j}^{n}-u_{i, j}^{n}}{h} \\
\nabla_{y}^{+} u_{i, j}^{n}=\frac{u_{i, j}^{n}-u_{i, j}^{n}}{h}, \quad \nabla_{y}^{-} u_{i, j}^{n}=\frac{u_{i, j-1}^{n}-u_{i, j}^{n}}{h} \\
\nabla_{x}^{+}\left[\Phi\left(u_{i, j}^{n}\right)\right]=\left(2-2 K\left(u_{i+1, j}^{n}, u_{i, j}^{n}\right)\right)^{1 / 2}, \\
\nabla_{x}^{-}\left[\Phi\left(u_{i, j}^{n}\right)\right]=\left(2-2 K\left(u_{i-1, j}^{n}, u_{i, j}^{n}\right)\right)^{1 / 2}, \\
\nabla_{y}^{+}\left[\Phi\left(u_{i, j}^{n}\right)\right]=\left(2-2 K\left(u_{i, j+1}^{n}, u_{i, j}^{n}\right)\right)^{1 / 2}, \\
\nabla_{y}^{-}\left[\Phi\left(u_{i, j}^{n}\right)\right]=\left(2-2 K\left(u_{i, j-1}^{n}, u_{i, j}^{n}\right)\right)^{1 / 2} .
\end{gathered}
$$

Third, the gradient kernel $\left\|\nabla \Phi\left(u^{n}\right)\right\|$ is estimated using (19) and $c\left(\left\|\nabla \Phi\left(u^{n}\right)\right\|\right)$ is calculated. Then we calculate the following function:

$$
\begin{aligned}
g_{i, j}^{n} & =c\left(\left\|\nabla \Phi u_{i, j}^{n}\right\|\right) \nabla u_{i, j}^{n}, \\
\delta u_{i, j}^{n} & =\frac{u_{i, j}^{n}-u_{i, j}^{n-1}}{\Delta t}, \quad \delta^{2} u_{i, j}^{n}=\frac{\delta u_{i, j}^{n}-\delta u_{i, j}^{n-1}}{\Delta t} .
\end{aligned}
$$

The discrete explicit scheme of the (16) could be written as

$$
\begin{aligned}
\delta^{2} u_{i, j}^{n+1}+\lambda \delta u_{i, j}^{n+1}= & c\left(\nabla_{x}^{+}\left[\Phi\left(u_{i, j}^{n}\right)\right]\right) \nabla_{x}^{+} u_{i, j}^{n} \\
& +c\left(\nabla_{x}^{-}\left[\Phi\left(u_{i, j}^{n}\right)\right]\right) \nabla_{x}^{-} u_{i, j}^{n} \\
& +c\left(\nabla_{y}^{+}\left[\Phi\left(u_{i, j}^{n}\right)\right]\right) \nabla_{y}^{+} u_{i, j}^{n} \\
& +c\left(\nabla_{y}^{-}\left[\Phi\left(u_{i, j}^{n}\right)\right]\right) \nabla_{y}^{-} u_{i, j}^{n}, \\
u_{i, j}^{0}=u_{i, j}^{-1} & =u_{i, j}, \quad 1 \leq i \leq M, \quad 1 \leq j \leq N .
\end{aligned}
$$

Through the above equation, we can obtain $u_{i, j}^{1}$ by $u_{i, j}^{-1}$ and $u_{i, j}^{0}$. The program will be iterative until it satisfies the stopping condition.

\section{Experiment Results and Discussion}

In this section, we describe the experiment results to assess the performance of the proposed method. The performance of the proposed method is compared with that of PDE anisotropic diffusion (PM model), KAD method, TDE method, and ITDE method. We implement the proposed algorithm using Matlab 7.10.0. We tested on some test images with additive Gaussian white noise of different levels. The suggestive parameters are set to maximize the performance of the filters. So for different test images, the parameters are different. The most commonly used diffusivity function in anisotropic diffusion is the one in (4). So in our experiments, we select (18) as the diffusivity function in our method and select (4) in other compared methods. The radialbasis function kernel is used for its generalization ability and computational simplicity although other kernels with

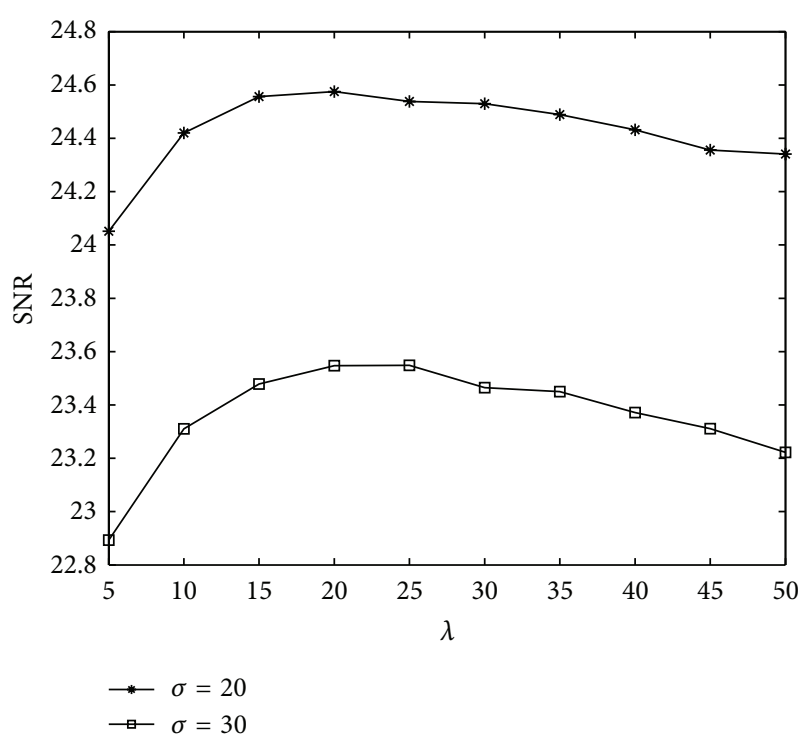

FIGURE 1: Comparison of different $\lambda$ on "lena" image.

appropriate parameter setting can also produce satisfactory results. In order to guarantee the stability of the numerical solver, the time step $d t$ should be small enough. However, if $d t$ is too small, the number of iterations for convergence will increase, which makes the method time consuming. We choose $\Delta t$ equals 0.1 in all diffusions. For the kernel based method including KAD and the proposed method, the kernel width $\sigma$ in (12) is chosen by the "trial-and-error" [22] technique. A wide range of $\sigma$ values can produce steady results. We select $\sigma$ ranging from 8 to 15 according to different noise levels and different images in our experiments. The diffusion stops when MAE is less than 0.005 .

We first discuss the effects of damping coefficient $\lambda$. From the numerical implementation model of this algorithm, the iterative equation can be written as

$$
u_{i, j}^{n}=\left(\frac{1}{1+\lambda \Delta t}\right)\left[(2+\lambda \Delta t) u_{i, j}^{n-1}-u_{i, j}^{n-2}+\Delta t^{2} \nabla g_{i, j}^{n-1}\right] \text {. }
$$

It is interesting to note that when $\lambda$ is large, (16) converges to the KAD algorithm after a very long time. When $\lambda$ is small, it may diffuse fast and lead to edge being smoothed. In order to objectively evaluate the performance of the different algorithms, we use signal-to-noise ratio (SNR), which is defined as follows:

$$
\mathrm{SNR}=10 \log _{10}\left(\frac{\sum_{i, j}\left(u_{i, j}^{0}\right)^{2}}{\sum_{i, j}\left(u_{i, j}^{0}-u_{i, j}\right)^{2}}\right) d B,
$$

where $u^{0}$ is the original noiseless image and $u$ denotes the recovered image.

Figure 1 shows the SNR value of filtered Lena image on different $\lambda$ value. We used noisy Lena image with different noise level $\widehat{\sigma}=20$ and $\widehat{\sigma}=30$, respectively. We select $\lambda$ from 5 to 50 and find that $\lambda$ ranging from 10 to 20 is appropriate for 


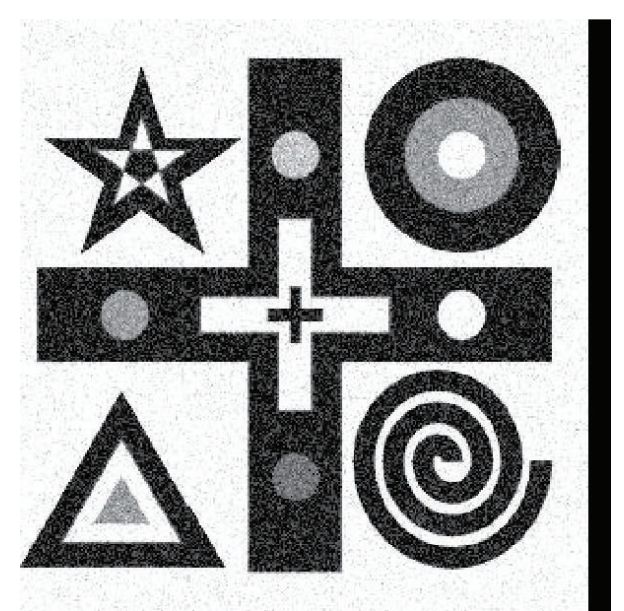

(a)

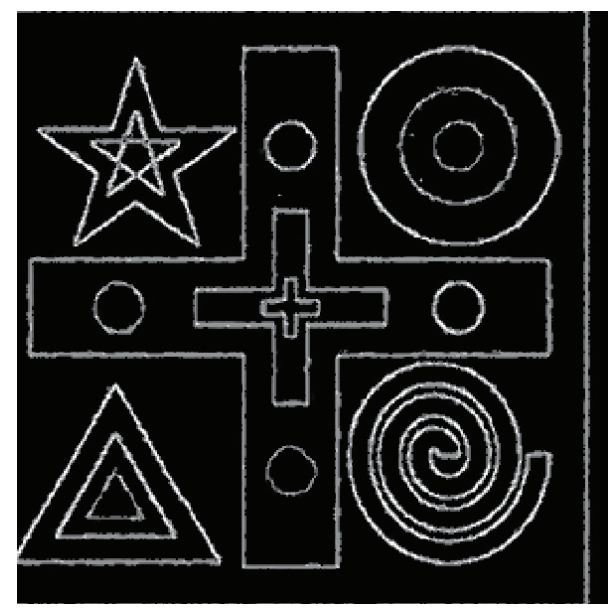

(b)

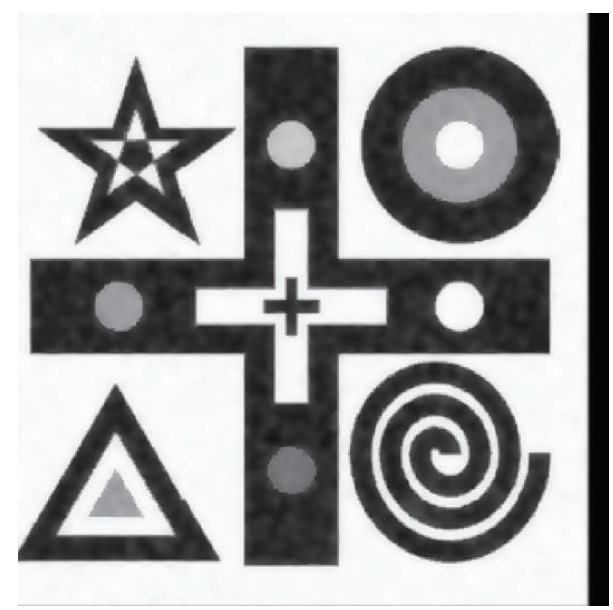

(c)

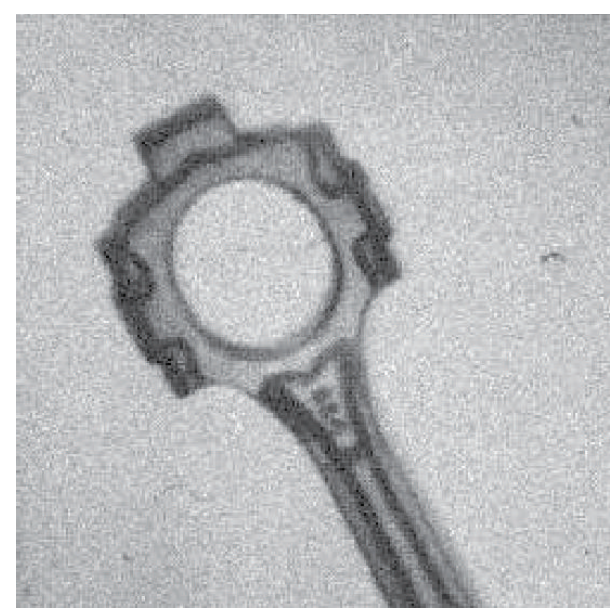

(d)

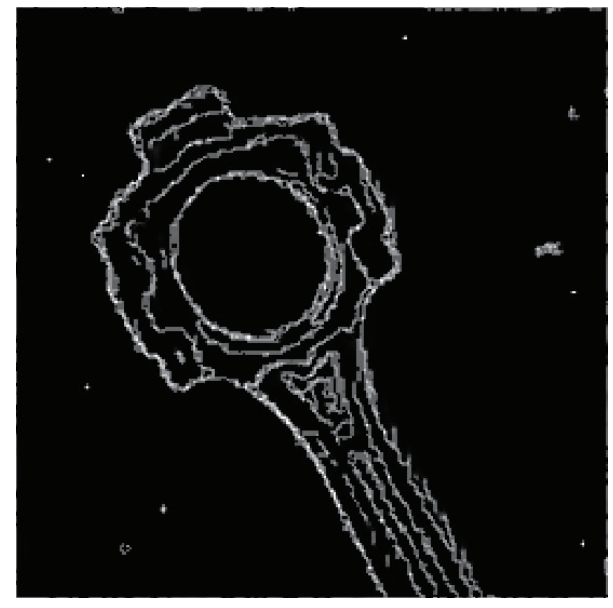

(e)

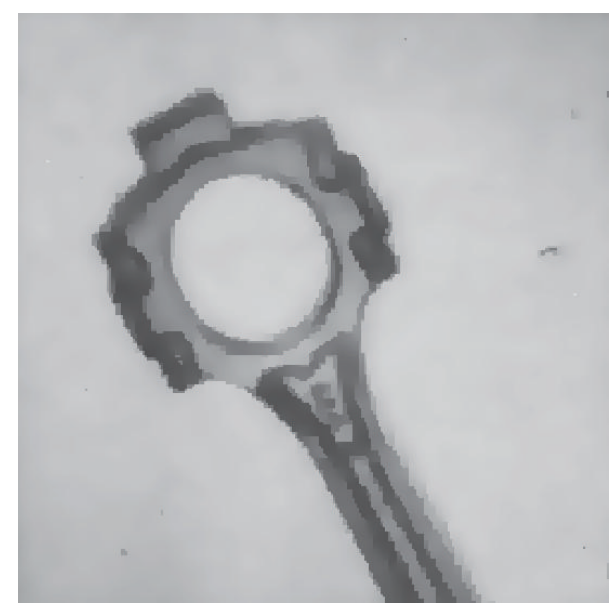

(f)

FIGURE 2: Edge detection and noise removal on synthetic images.

our method. When it is larger than 20, there are no notable improvements in visual effect and SNR improvement but it leads to time consumption. When $\lambda$ is between 10 and 20 , the algorithm converges faster. Image noise can be removed and edges are preserved. So in most of our experiments, we select $\lambda$ ranging from 10 to 20 for computational convenience.

Figure 2 shows the proposed method on synthetic image for edge detection and noise removal. It can be found that the 


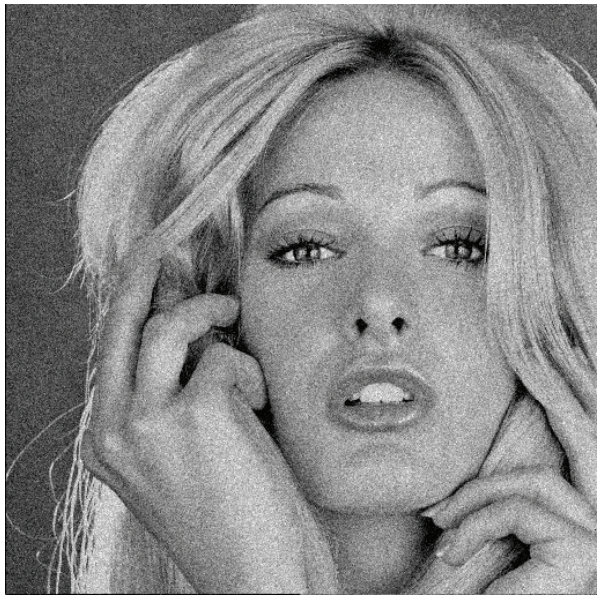

(a)

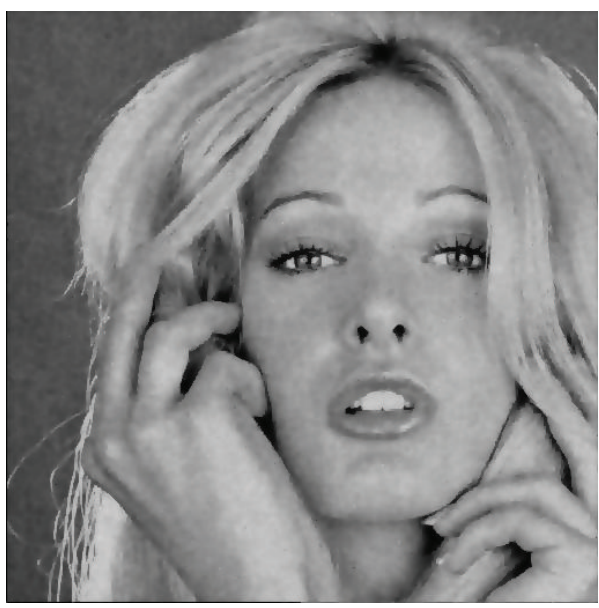

(c)

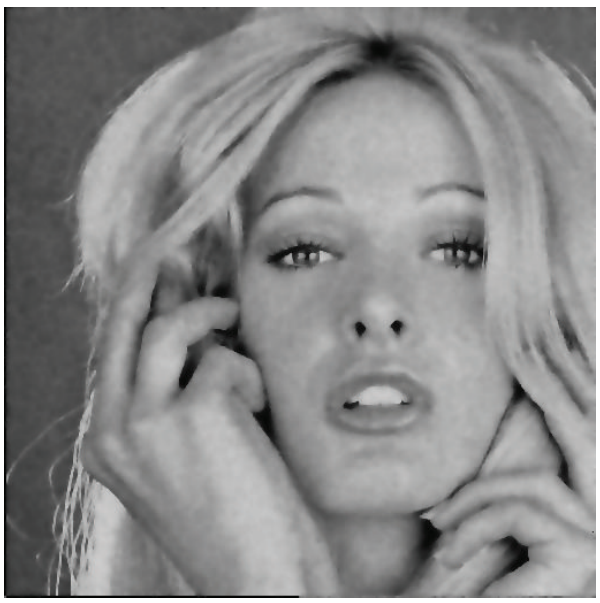

(e)

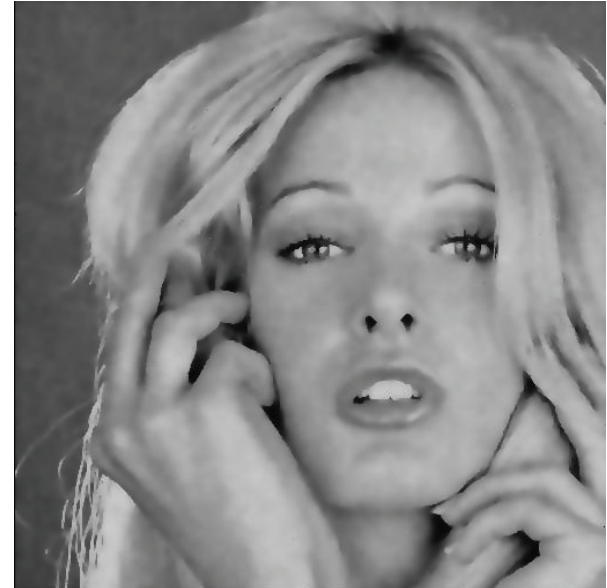

(b)

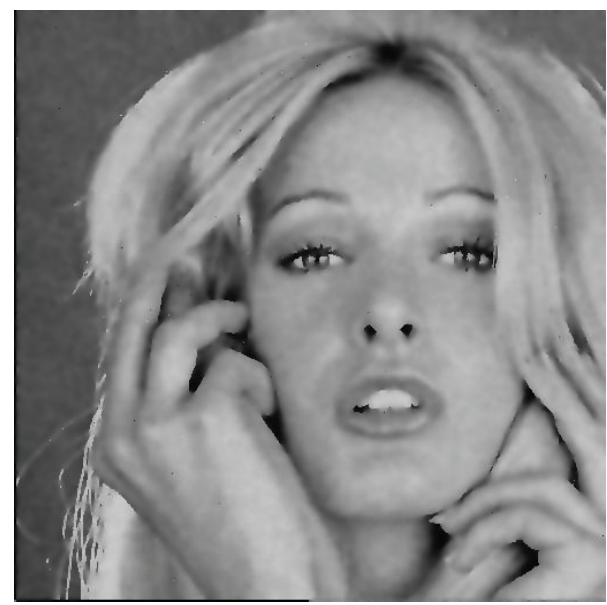

(d)

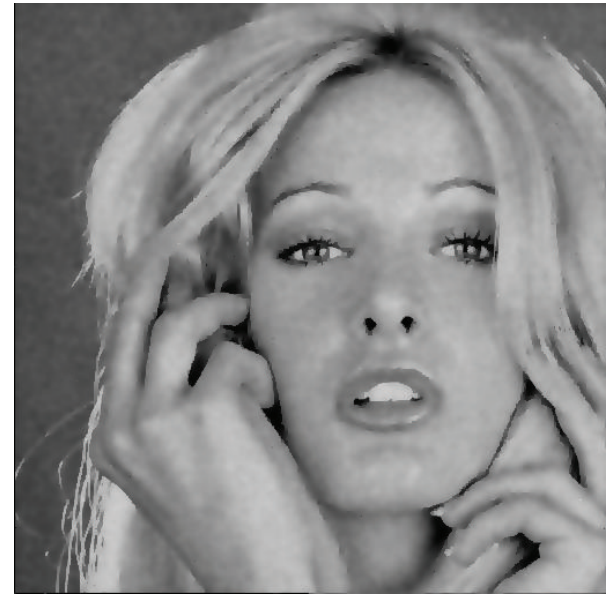

(f)

FIgURE 3: Comparison of different methods on "Tiffany" image. (a) The noisy image, $\widehat{\sigma}=20$, (b) PM diffusion, (c) KAD method, (d) TDE, (e) ITDE, and (f) the proposed KTDE. 


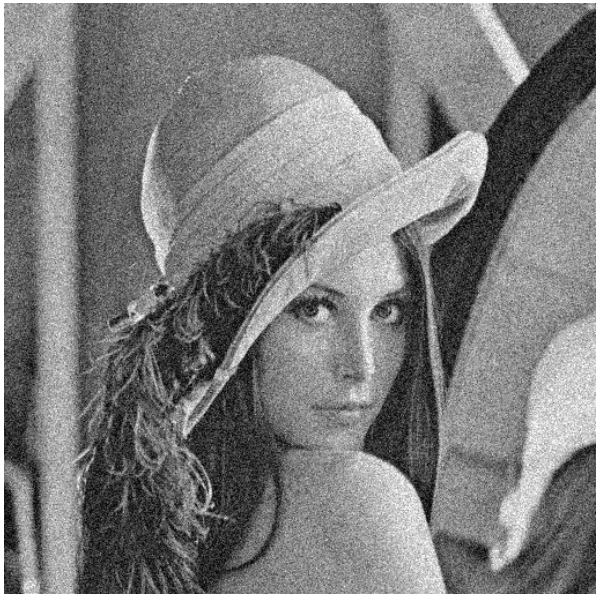

(a)

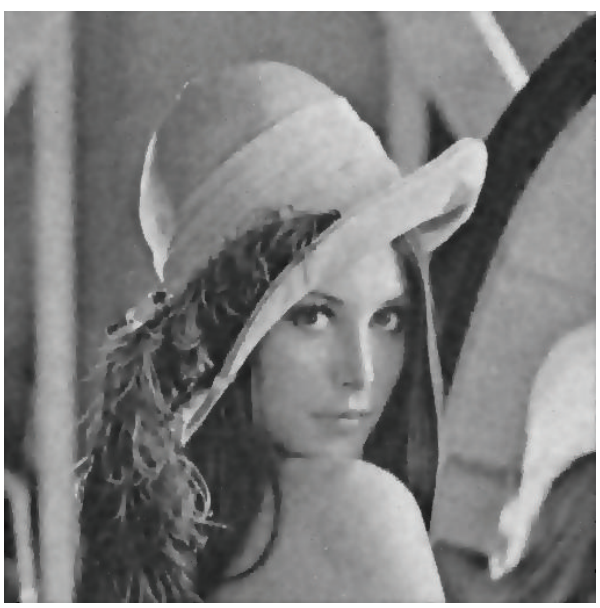

(c)

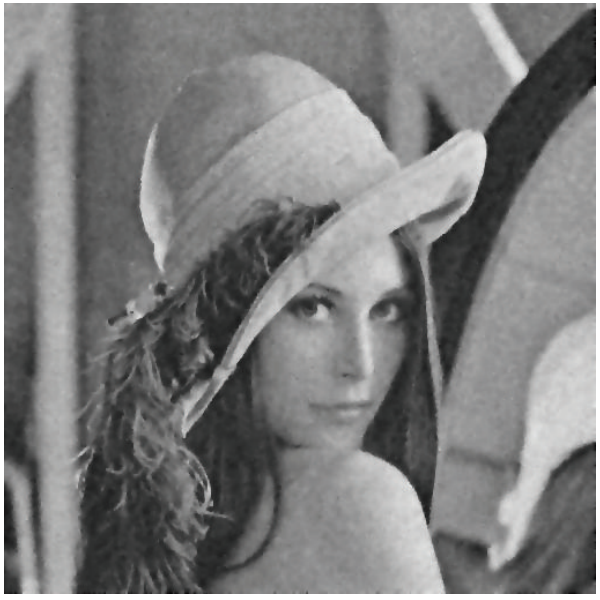

(e)

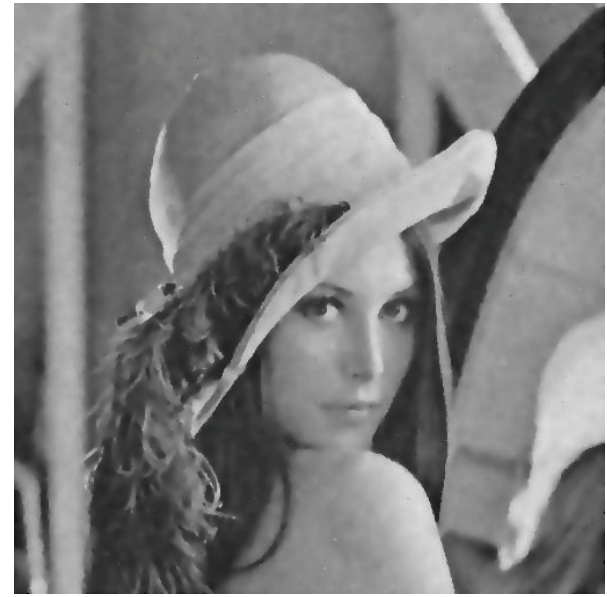

(b)

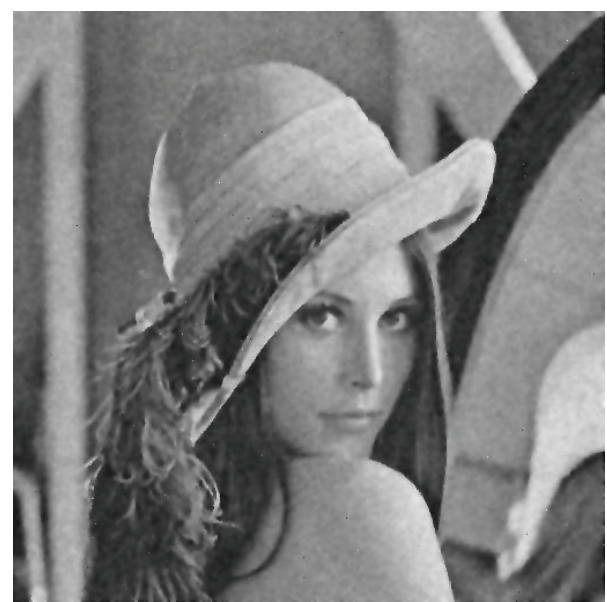

(d)

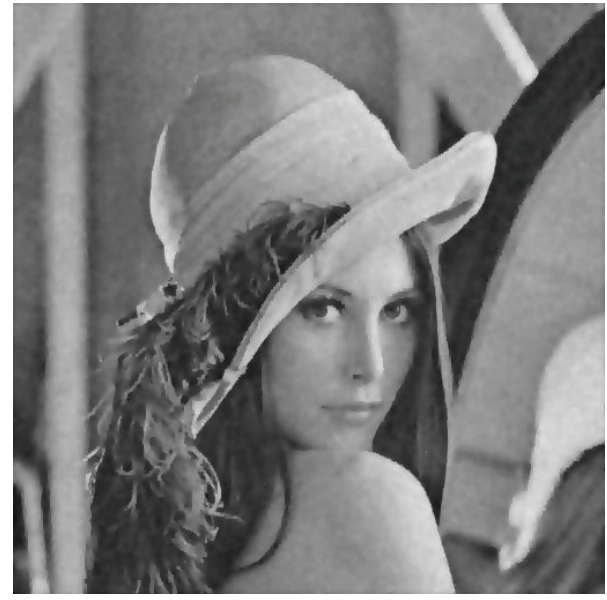

(f)

Figure 4: Comparison of different methods on "Lena" image. (a) The noisy image, $\widehat{\sigma}=25$, (b) PM diffusion, (c) KAD method, (d) TDE, (e) ITDE, and (f) the proposed KTDE. 


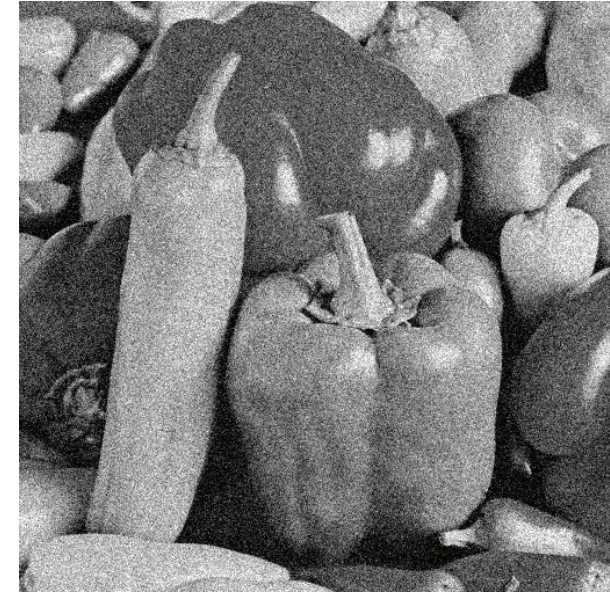

(a)

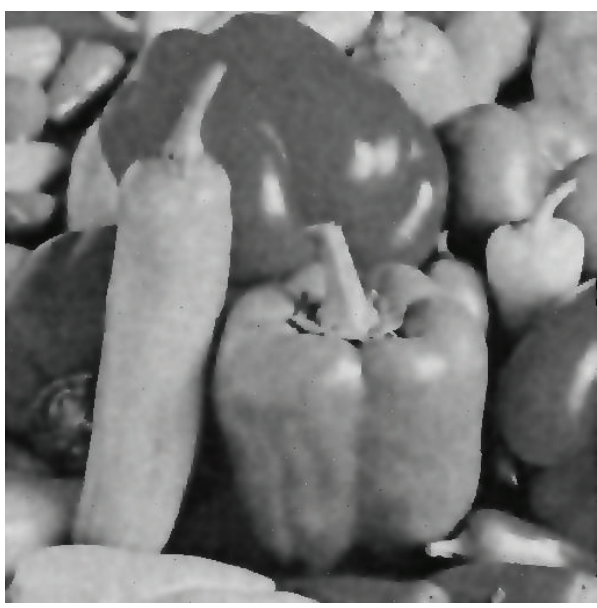

(c)

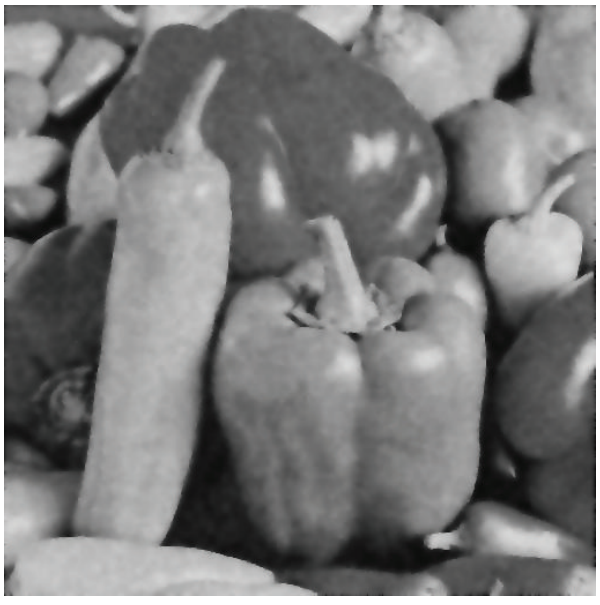

(e)

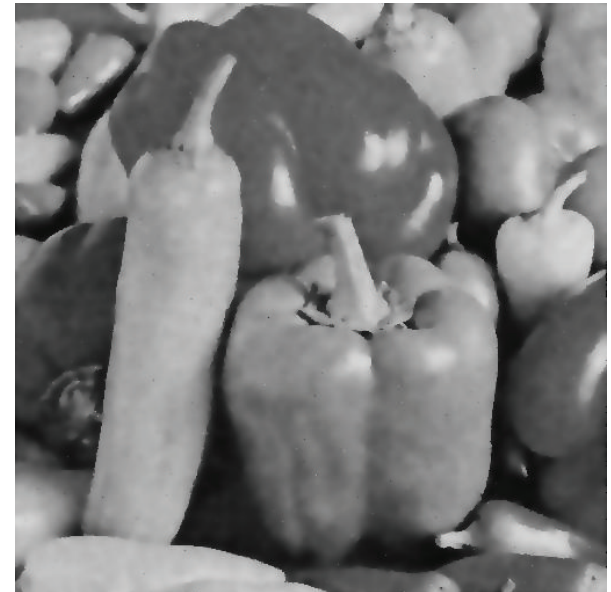

(b)

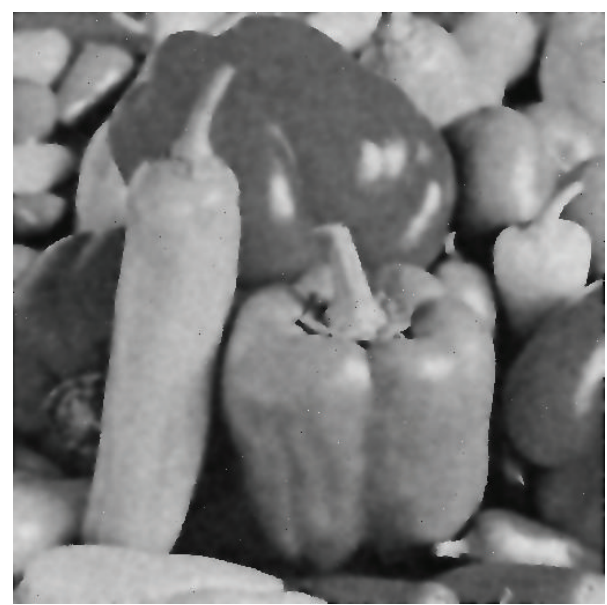

(d)

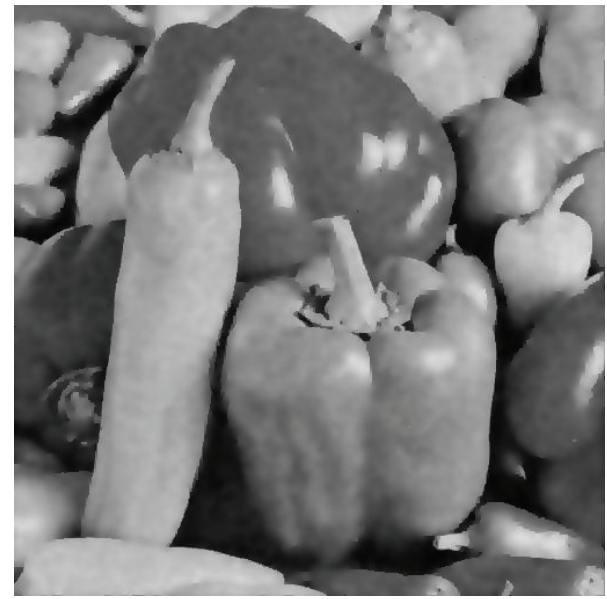

(f)

FIGURE 5: Comparison of different methods on "peppers" image. (a) The noisy image, $\widehat{\sigma}=30$, (b) PM diffusion, (c) KAD method, (d) TDE, (e) ITDE, and (f) the proposed KTDE. 
TABLE 1: The comparison of SNR on noisy images with Gaussian white noise $\widehat{\sigma}=20$.

\begin{tabular}{lccccc}
\hline Method & PM & KAD & TDE & ITDE & 24.2727 \\
\hline Lena & 24.0680 & 24.3613 & 24.0961 & 23.5653 & 24.5754 \\
Tiffany & 23.3850 & 23.5351 & 23.4762 & 25.0878 & 24.2414 \\
House & 25.0831 & 25.8424 & 24.7632 & 20.9658 & 25.8611 \\
Barbara & 20.6853 & 20.4471 & 20.0258 & 25.4822 \\
Peppers & 24.7149 & 25.2177 & 24.7121 & 21.2630 \\
\hline
\end{tabular}

TABLE 2: The comparison of SNR on noisy images with Gaussian white noise $\widehat{\sigma}=30$.

\begin{tabular}{lccccc}
\hline Method & PM & KAD & TDE & ITDE & 23.3016 \\
Lena & 23.0347 & 23.2775 & 22.8559 & 22.7838 & 23.5468 \\
Tiffany & 22.5260 & 22.6469 & 21.3544 & 23.8953 & 24.8834 \\
House & 23.7300 & 23.8632 & 22.5367 & 19.1953 & 20.7627 \\
Barbara & 18.7728 & 18.8927 & 18.4283 & 23.4351 & 24.3382 \\
Peppers & 23.3754 & 23.6147 & 22.2105 & & \\
\hline
\end{tabular}

proposed method has good performance on edge detection and noise removal.

To verify the effectiveness of our proposed kernel based TDE (KTDE) method for image denoising, it was evaluated in comparison with PM second PDE method [1], KAD method [22], TDE method [17], and ITDE method [21]. In Figure 3, the denoising effects of these methods are tested on a standard digital image. We add Gaussian white noise in Figure 3(a), which is independently and identically distributed (i.i.d) Gaussian real variables. Figures 3(b)-3(f) show the denoised image of different methods. It is obvious that our method has better capability on noise removal and edge preservation. Our method does not cause blocky effect and spots. Meanwhile, the edges and important textures are well preserved. We added a heavy Gaussian white noise in Figures 4(a) and 5(a), and the results yielded by PM anisotropic diffusion equation and the KAD method, TDE method, and ITDE method are shown in Figures 4(b)-4(e) and Figures 5(b)-5(e). The results of our method are shown in Figures 4(f) and 5(f). They leave more sharp edges than the other four methods while the noise can be suppressed efficiently. We can conclude that our method has better performance on low SNR images.

In Tables 1 and 2, the comparison between the performances of these filters in terms of SNR of the denoised images is presented. The proposed method gives the denoised images higher SNR. The good performance of the proposed method is apparent. So the proposed method has better performance in noise removal compared with the other PDE methods.

\section{Conclusion}

A kernel based telegraph-diffusion equation to suppress image noise is proposed in this paper. When images are corrupted by heavy noise, the relationships between edges and noise are transformed to linearly separable ones by kernel function. This kernel based telegraph-diffusion method improves the effectiveness of the diffusion method in denoising low SNR images. It exhibits superior performance in both noise reduction and edge localization. Experiment results have shown that this new method outperforms original anisotropic diffusion method, kernel anisotropic diffusion method, and the original telegraph-diffusion method. Fine details, sharp corners, and thin lines are preserved. The proposed method inherits the advantage of telegraph-diffusion and kernel function. It can be used for preprocessing step for further image processing.

\section{Conflict of Interests}

The authors declare that there is no conflict of interests regarding the publication of this paper.

\section{Acknowledgments}

The authors would like to thank the anonymous referees for their valuable comments and suggestions, which actually stimulated this work. This work was supported by the National Natural Science Foundation of China (Grant nos. 61201297, 11101320, and 11201362).

\section{References}

[1] P. Perona and J. Malik, "Scale-space and edge detection using anisotropic diffusion," IEEE Transactions on Pattern Analysis and Machine Intelligence, vol. 12, no. 7, pp. 629-639, 1990.

[2] L. I. Rudin, S. Osher, and E. Fatemi, "Nonlinear total variation based noise removal algorithms," Physica D: Nonlinear Phenomena, vol. 60, no. 1-4, pp. 259-268, 1992.

[3] B. B. Kimia, A. Tannenbaum, and S. W. Zucker, "On the evolution of curves via a function of curvature. I: the classical case," Journal of Mathematical Analysis and Applications, vol. 163, no. 2, pp. 438-458, 1992.

[4] F. Catté, P. L. Lions, J. M. Morel, and T. Coll, "Image selective smoothing and edge detection by nonlinear diffusion," SIAM Journal on Numerical Analysis, vol. 29, no. 1, pp. 182-193, 1992.

[5] H. Romeny and M. Bart, Eds., Geometry-Driven Diffusion in Computer Vision, Kluwer Academic, Norwell, Mass, USA, 1994.

[6] J. Weickert, Anisotropic Diffusion in Image Processing, Teubner, Stuttgart, Germany, 1998. 
[7] T. F. Chan, J. Shen, and L. Vese, "Variational PDE models in image processing," Notices of The American Mathematical Society, vol. 50, no. 1, 2003.

[8] B. Ham, D. Min, and K. Sohn, "Robust scale-space filter using second-order partial differential equations," IEEE Transactions on Image Processing, vol. 21, no. 9, pp. 3937-3951, 2012.

[9] Z. Gui, Y. Liu, and J. He, "PML algorithm for positron emission tomography combined with nonlocal fuzzy anisotropic diffusion filtering," IEEE Transactions on Nuclear Science, vol. 59, no. 5, pp. 1984-1989, 2012.

[10] Q. Chen, P. Montesinos, Q. S. Sun, and D. Shen Xia, "Ramp preserving Perona-Malik model," Signal Processing, vol. 90, no. 6, pp. 1963-1975, 2010.

[11] Y. L. You and M. Kaveh, "Fourth-order partial differential equations for noise removal," IEEE Transactions on Image Processing, vol. 9, no. 10, pp. 1723-1730, 2000.

[12] M. Lysaker, A. Lundervold, and X. C. Tai, "Noise removal using fourth-order partial differential equation with applications to medical magnetic resonance images in space and time," IEEE Transactions on Image Processing, vol. 12, no. 12, pp. 1579-1590, 2003.

[13] F. Li, C. Shen, J. Fan, and C. Shen, "Image restoration combining a total variational filter and a fourth-order filter," Journal of Visual Communication and Image Representation, vol. 18, no. 4 , pp. 322-330, 2007.

[14] J. Rajan, K. Kannan, and M. R. Kaimal, "An improved hybrid model for molecular image denoising," Journal of Mathematical Imaging and Vision, vol. 31, no. 1, pp. 73-79, 2008.

[15] Y. Wang, X. Ji, and Q. Dai, "Fourth-order oriented partialdifferential equations for noise removal of two-photon fluorescence images," Optics Letters, vol. 35, no. 17, pp. 2943-2945, 2010.

[16] M. R. Hajiaboli, "An anisotropic fourth-order diffusion filter for image noise removal," International Journal of Computer Vision, vol. 92, no. 2, pp. 177-191, 2011.

[17] V. Ratner and Y. Y. Zeevi, "Image enhancement using elastic manifolds," in Proceeding of the 14th International Conference on Image Analysis and Processing (ICIAP '07), pp. 769-774, IEEE Computer Society, Modena, Italy, September 2007.

[18] W. Zeng, X. Lu, and X. Tan, "Non-linear fourth-order telegraphdiffusion equation for noise removal," IET Image Processing, vol. 7, no. 4, pp. 335-342, 2013.

[19] Y. L. You, W. Xu, A. Tannenbaum, and K. Mostafa, "Behavioral analysis of anisotropic diffusion in image processing," IEEE Transactions on Image Processing, vol. 5, no. 11, pp. 1539-1553, 1996.

[20] J. Weickert, "A review of nonlinear diffusion filtering," in ScaleSpace Theory in Computer Vision, pp. 1-28, Springer, Berlin, Germany, 1997.

[21] Y. Cao, J. Yin, Q. Liu, and M. Li, "A class of nonlinear parabolichyperbolic equations applied to image restoration," Nonlinear Analysis: Real World Applications, vol. 11, no. 1, pp. 253-261, 2010.

[22] J. Yu, Y. Wang, and Y. Shen, "Noise reduction and edge detection via kernel anisotropic diffusion," Pattern Recognition Letters, vol. 29, no. 10, pp. 1496-1503, 2008.

[23] A. H. Shabani, J. S. Zelek, and D. A. Clausi, "Regularized gradient kernel anisotropic diffusion for better image filtering," in Proceeding of the 9th Conference on Computer and Robot Vision (CRV'12), pp. 383-387, IEEE, Toronto, Canada, May 2012.
[24] F. Zhang, Y. M. Yoo, L. M. Koh, and Y. Kim, "Nonlinear diffusion in laplacian pyramid domain for ultrasonic speckle reduction," IEEE Transactions on Medical Imaging, vol. 26, no. 2, pp. 200211, 2007. 


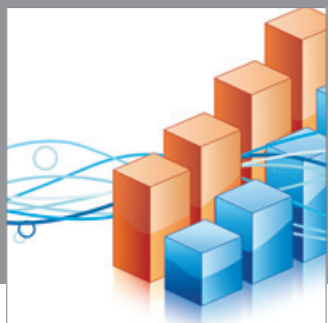

Advances in

Operations Research

mansans

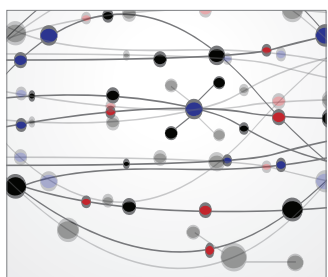

The Scientific World Journal
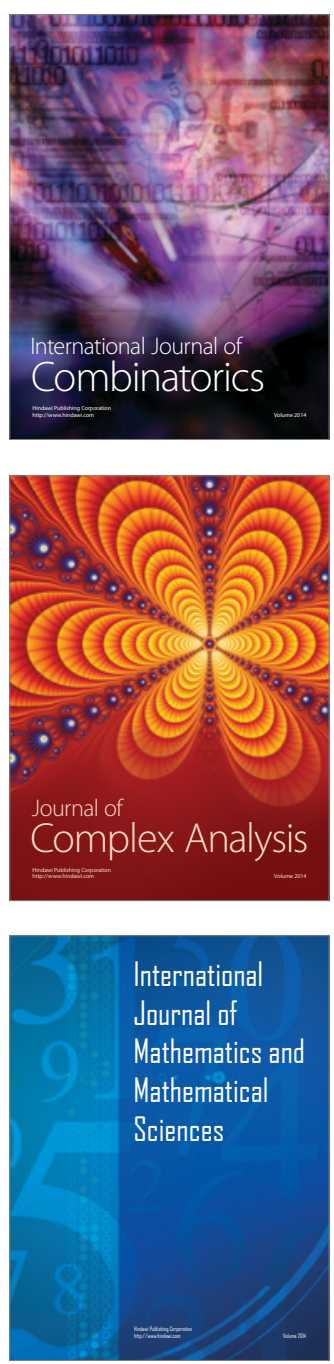
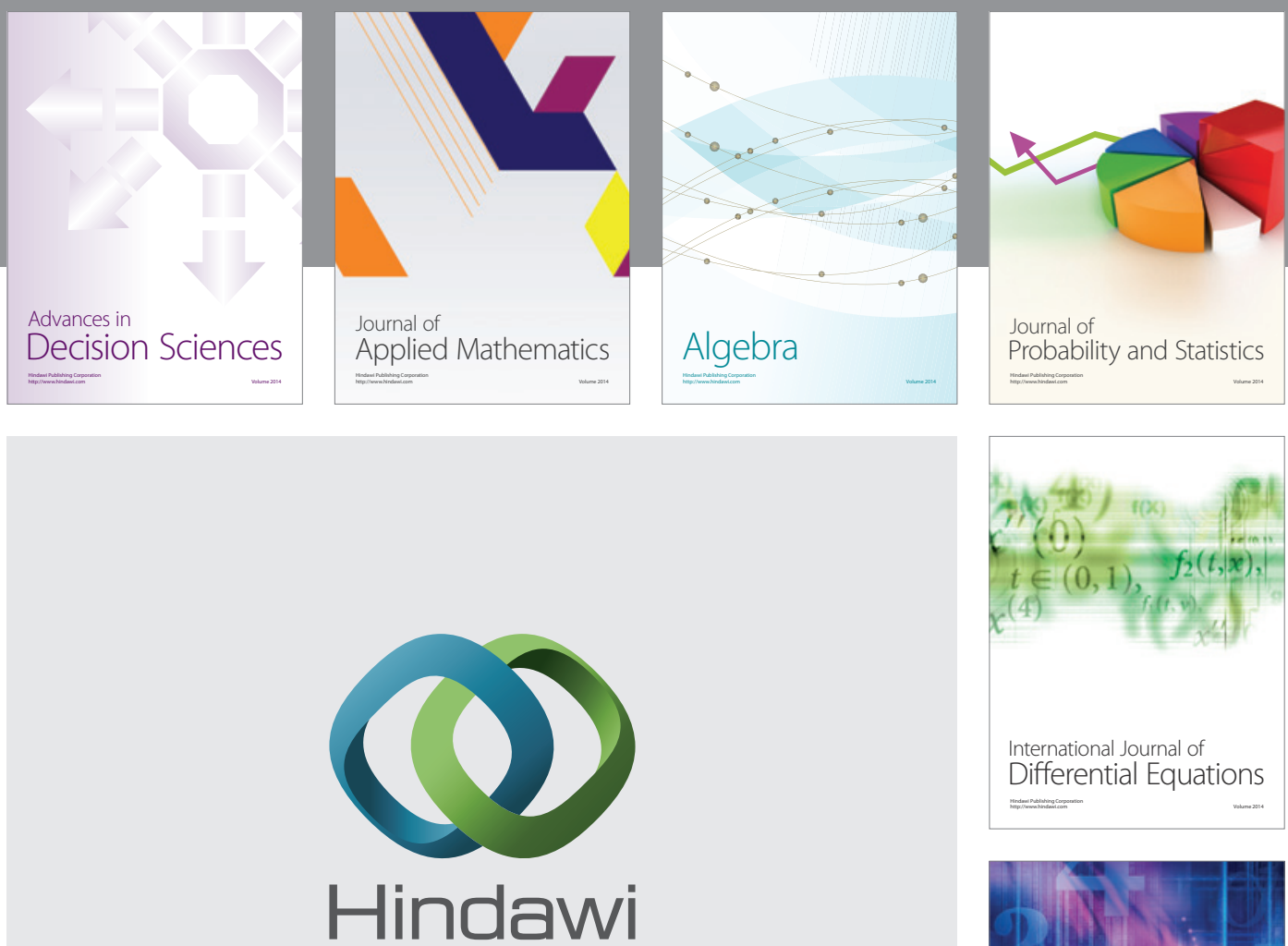

Submit your manuscripts at http://www.hindawi.com
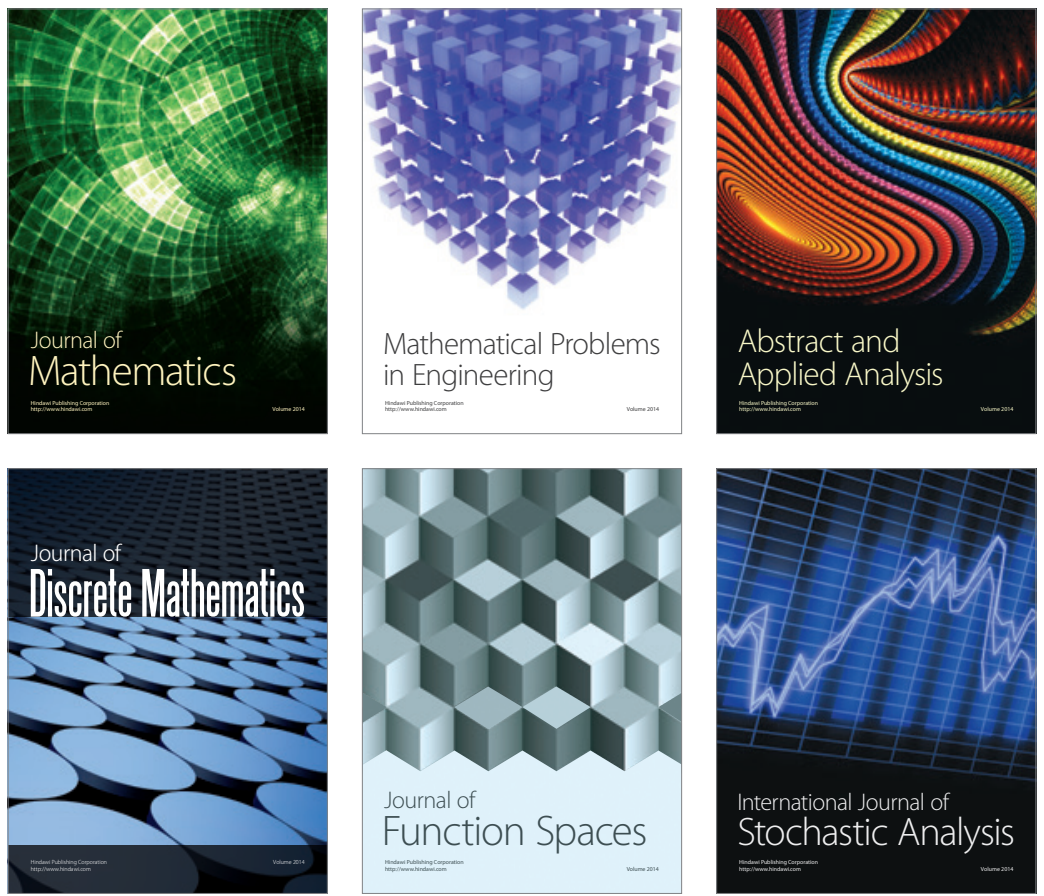

Journal of

Function Spaces

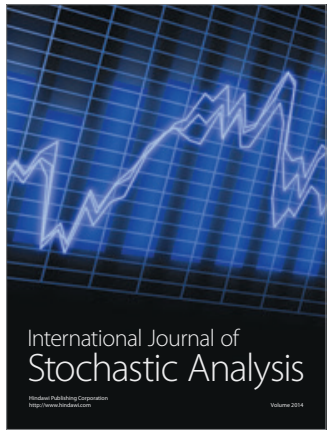

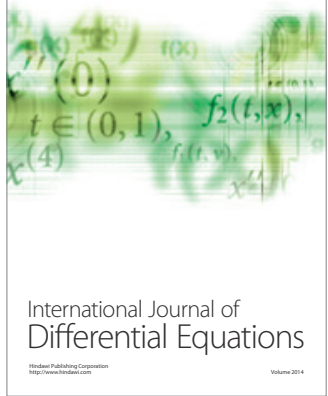
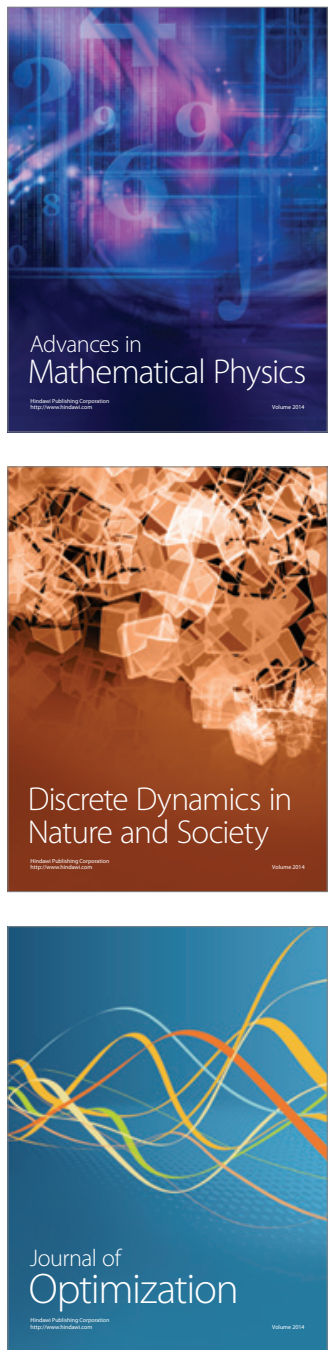